\title{
Soleus fiber force and maximal shortening velocity after non-weight bearing with intermittent activity
}

\author{
JEFFREY J. WIDRICK, JILL J. BANGART, MILOSLAV KARHANEK, AND ROBERT H. FITTS \\ Department of Biology, Marquette University, Milwaukee, Wisconsin 53201
}

Widrick, Jeffrey J., Jill J. Bangart, Miloslav Karhanek, and Robert $H$. Fitts. Soleus fiber force and maximal shortening velocity non-weight bearing with intermittent activity. J. Appl. Physiol. 80(3): 981-987, 1996.-This study examined the effectiveness of intermittent weight bearing (IWB) as a countermeasure to non-weight-bearing (NWB)-induced alterations in soleus type I fiber force (in $\mathrm{mN}$ ), tension ( $\mathrm{P}_{\mathrm{a}}$; force per fiber cross-sectional area in $\left.\mathrm{kN} / \mathrm{m}^{-2}\right)$, and maximal unloaded shortening velocity $\left(V_{0}\right.$, in fiber lengths/s). Adult rats were assigned to one of the following groups: normal weight bearing (WB), 14 days of hindlimb NWB (NWB group), and 14 days of hindlimb NWB with IWB treatments (IWB group). The IWB treatment consisted of four 10-min periods of standing WB each day. Single, chemically permeabilized soleus fiber segments were mounted between a force transducer and position motor and were studied at maximal $\mathrm{Ca}^{2+}$ activation, after which type I fiber myosin heavy-chain composition was confirmed by sodium dodecyl sufate-polyacrylamide gel electrophoresis. NWB resulted in a loss in relative soleus mass $(-45 \%)$, with type I fibers displaying reductions in diameter $(-28 \%)$ and peak isometric force $(-55 \%)$ and an increase in $V_{0}(+33 \%)$. In addition, NWB induced a $16 \%$ reduction in type $I$ fiber $P_{0}$, a $41 \%$ reduction in type I fiber peak elastic modulus [ $\mathrm{E}_{\mathrm{o}}$, defined as $(\Delta$ force/ $\Delta$ length $) \times$ (fiber length/fiber cross-sectional area] and a significant increase in the $P_{0} / E_{0}$ ratio. In contrast to NWB, IWB reduced the loss of relative soleus mass (by 22\%) and attenuated alterations in type I fiber diameter (by $36 \%$ ), peak force (by $29 \%$ ), and $V_{\mathrm{o}}$ (by $48 \%$ ) but had no significant effect on $\mathrm{P}_{0}, \mathrm{E}_{\mathrm{o}}$, or $\mathrm{P}_{0} / \mathrm{E}_{\mathrm{o}}$. These results indicate that a modest restoration of WB activity during 14 days of NWB is sufficient to attenuate type I fiber atrophy and to partially restore type I peak isometric force and $V_{0}$ to WB levels. However, the NWB-induced reductions in $\mathrm{P}_{0}$ and $\mathrm{E}_{\mathrm{o}}$, which we hypothesize to be due to a decline in the number and stiffness of cross bridges, respectively, are considerably less responsive to this countermeasure treatment.

muscle atrophy; hindlimb suspension; hindlimb unloading; countermeasures

THE ABSENCE OF NORMAL weight-bearing (WB) activity during spaceflight produces rapid alterations in antigravity muscle mass and function. After exposure to as little as 4-7 days of zero gravity, the rodent soleus exhibits a $20-37 \%$ reduction in mass, a parallel decline in force production, and a significant increase in fatigability (1, 17, 20). Similarly, human crew members returning from extended spaceflight display atrophy, weakness, and increased fatigability of the antigravity muscles of the lower limbs (2). These observations underscore the necessity of designing and implementing in-flight countermeasures to prevent detrimental changes in muscle mass and function during longduration space missions.
Rodent hindlimb suspension is often used as a groundbased model with which to study non-weight-bearing (NWB) muscle atrophy because it produces changes in muscle mass and function that are quantitatively similar to those observed after spaceflight $(1,5,7,16,17,20$, 29,31 ). This model has recently been used to test the effectiveness of various interventions designed to prevent or attenuate antigravity muscle atrophy during NWB. Perhaps the simplest and most straightforward of these countermeasures has been to provide intermittent periods of weight support over the course of NWB. Four brief sessions (10-15 min/session) of weight support each day during 7 days of NWB preserved soleus wet weight $(3,26)$, whereas a single daily session of either 2 or $4 \mathrm{~h}$ of weight support reduced soleus atrophy by $\sim 50 \%$ over a 28 -day period of NWB (31). Furthermore, intermittent weight support prevented the decline in soleus peak isometric force observed after 7 days of NWB (26). These results indicate that some form of intermittent weight bearing (IWB) may be an effective countermeasure to NWB-induced muscle atrophy. During spaceflight, when normal WB is impossible, centrifugation may prove to be an alternative method of "reloading" antigravity muscles $(3,4)$.

A major aim of this laboratory is to understand how NWB alters cross-bridge mechanisms of muscle contraction. Studies performed on single skinned type I soleus fibers indicate that the reduction in force after NWB is not only due to simple fiber atrophy but also to a reduction in fiber tension $\left(\mathrm{P}_{0}\right)$, or the force produced per unit cross-sectional area (CSA) of the fiber $(8,21,22)$. Recent measurements of peak elastic modulus $\left(E_{0}\right)$, which is thought to reflect the relative number of actomyosin cross-bridge attachments per CSA (15), indicate that this reduction in $\mathrm{P}_{0}$ is due to fewer cross bridges per CSA after NWB (23). In addition to changes in force production, the majority of the fibers that express type I myosin heavy chain (MHC) after NWB also display an elevated maximal unloaded shortening velocity $\left(V_{0}\right)(8,22)$. Thus, in this population of fibers, there is an increase in the maximal cross-bridge cycling rate that appears to be dissociated from fiber $\mathrm{MHC}$ expression.

Although intermittent weight support has been shown to reduce the muscle atrophy induced by NWB, the extent to which this treatment may modulate alterations in cross-bridge mechanisms of contraction is unknown. Therefore, the purpose of this study was to examine the effects of IWB on peak force production and $V_{0}$ of permeabilized single type I soleus fibers after a 14-day period of NWB. 


\section{METHODS}

Animals. Male Sprague-Dawley rats were assigned to the following treatment groups: caged WB (WB group), 14 days of hindlimb NWB (NWB group), and 14 days of hindlimb NWB with IWB treatments (IWB group). All groups were maintained on a 12:12-h light-dark cycle, but the WB rats were housed separately from the NWB and IWB animals to minimize spontaneous activity (7). All rats had ad libitum access to chow and tap water.

Experimental treatments. NWB and IWB rats were suspended from a tail harness, as previously described in detail (7). Briefly, vinyl cloth was glued to the proximal two-thirds of the tail, and the cloth and tail were wrapped together with elastic tape. After the glue dried, the rats were suspended above a horizontal grid floor by means of a fishing swivel that passed through the apex of a small triangular wire loop extending from the center portion of the vinyl cloth. The height of the fishing swivel was adjusted so that the hindlimbs of the rats were unable to contact the grid floor or any other supportive surface. Rats were able to use their forelimbs to gain access to food and water. The color of the exposed distal portion of the tail was closely monitored during suspension to ensure that the tail harness did not obstruct blood flow.

At $0,4,8$, and $11.75 \mathrm{~h}$ of the light cycle, the IWB rats were removed from suspension and placed in a standing position on the grid floor. A small wire enclosure was placed over the rats to prevent extensive walking activity. IWB rats were resuspended after 10 min of weight support.

Single-fiber preparation. Animals were anesthetized with pentobarbital sodium (50 $\mathrm{mg} / \mathrm{kg}$ body wt ip), and one soleus was removed, trimmed of excess fat and connective tissue, weighed, and placed in cold relaxing solution (for composition, see below). Small bundles 1 - to 2 -mm wide were formed from the muscle, pinned out at approximately in situ length, tied to small pieces of glass capillary tubes, and stored in skinning solution at $-20^{\circ} \mathrm{C}$ for up to $4 \mathrm{wk}$. The skinning solution contained (in $\mathrm{mM}$ ) $125 \mathrm{~K}$-propionate, 20.0 imidazole (pH 7.0), 2.0 ethylene glycol-bis ( $\beta$-aminoethyl ether)- $N, N, N^{\prime}$, $N^{\prime}$-tetraacetic acid (EGTA), $4.0 \mathrm{ATP}, 1.0 \mathrm{MgCl}_{2}$, and $50 \%$ glycerol (vol/vol).

On the day of an experiment, a muscle bundle was placed in cold relaxing solution, and a single-fiber segment was isolated and transferred into a temperature-regulated experimental chamber. While under relaxing solution, the fiber ends were attached to small stainless steel troughs by using monofilament posts and 10-0 suture, as previously described in detail (25). One trough was connected to a force transducer (Cambridge model 400; Cambridge Technology, Watertown, MA) and the other to a servo-controlled position motor (Cambridge model 300B; Cambridge Technology). The experimental chamber was mounted on an inverted microscope stage and arranged so that it was possible to rapidly transfer the mounted fiber into an adjacent chamber containing activating solution (for composition, see below). Sarcomere spacing and uniformity could be monitored in both relaxing and activating solutions by viewing the fiber at $\times 800$ through the glass bottoms of each chamber. Once mounted, the fiber was briefly bathed in relaxing solution containing $0.5 \%$ polyoxyethylene 20 cetyl ether (Brij 58; Sigma Chemical, St. Louis, MO) to improve sarcomere resolution and ensure disruption of the sarcoplasmic reticulum.

Sarcomere spacing was adjusted to $2.5 \mu \mathrm{m}$. Fiber length (FL) was measured as the length of the fiber segment suspended between the two troughs and averaged $2.09 \pm 0.02$ (SE) $\mathrm{mm}$ for the 173 fibers examined in this study. A Polaroid photo was taken of the fiber while it was briefly suspended in air. Fiber width was determined as the mean of three measurements made at equal intervals along the length of the photo. Because the fiber rounds up when removed from solution, fiber CSA was calculated by equating the mean fiber width to fiber diameter, with the assumption of a circular CSA.

The composition of the relaxing and activating solutions was calculated by using the computer program of Fabiato and Fabiato (6) and the apparent stability constants reported by Godt and Lindley (10). The relaxing solution had a free $\mathrm{Ca}^{2+}$ concentration $\left(\left[\mathrm{Ca}^{2+}\right]\right.$ ) of pCa 9.0 (where pCa $=-\log \left[\mathrm{Ca}^{2+}\right]$ ) and contained (in $\mathrm{mM}$ ) 20.0 imidazole, 7.0 EGTA, 10.0 caffeine, $4.74 \mathrm{ATP}, 14.5$ creatine phosphate, $5.40 \mathrm{MgCl}_{2}$, and $0.016 \mathrm{CaCl}_{2} \cdot 2 \mathrm{H}_{2} \mathrm{O}$. The maximal activating solution, $\mathrm{pCa} 4.5$, contained (in mM) 20.0 imidazole, 7.0 EGTA, 10.0 caffeine, 4.81 ATP, 14.5 creatine phosphate, $5.26 \mathrm{MgCl}_{2}$, and 7.0 $\mathrm{CaCl}_{2} \cdot 2 \mathrm{H}_{2} \mathrm{O}$. Both solutions contained sufficient $\mathrm{KOH}$ and $\mathrm{KCl}$ to achieve a $\mathrm{pH}$ of 7.0 and a total ionic strength of 180 $\mathrm{mmol} / \mathrm{l}$.

The temperature of the activating solution was maintained at $15^{\circ} \mathrm{C}$ during all experiments. Unamplified outputs from the force transducer and position motor were monitored on a digital oscilloscope, amplified, and interfaced to an IBMcompatible PC via a Lab Master DMA input-output board (Scientific Solutions, Solon, $\mathrm{OH}$ ). Custom-designed software coordinated data collection, stored force and position data to disk, and performed the calculations described below.

Peak force measurements. Peak force (in $\mathrm{mN}$ ) was calculated as the difference between force recorded while the fiber was in relaxing solution and the peak force obtained during $\mathrm{Ca}^{2+}$ activation. $\mathrm{P}_{\mathrm{o}}$ (in $\mathrm{kN} / \mathrm{m}^{2}$ ) was defined as peak force divided by the CSA of the fiber.

Fiber $E_{\sigma}$ Peak $\mathrm{E}_{0}$ was determined from analysis of force and position transients obtained during rapid fiber oscillation, as previously described by McDonald and Fitts (23). While in relaxing solution, the fiber was briefly oscillated by driving the position motor with a $1.5-\mathrm{kHz}$ sine wave at an amplitude that elicited a $0.05 \%$ peak-to-peak change in FL. Once the fiber had attained peak isometric force, an identical sine wave was applied to the position motor. Force and position transients obtained in relaxing and activation solutions were directed to a peak-to-peak detector circuit, where they were converted into direct current voltage outputs ( $\Delta$ force and $\Delta$ length). To account for systematic contributions to stiffness measurements, adjusted $\Delta$ force and $\Delta$ length values were obtained by subtracting $\Delta$ force and $\Delta$ length measured in relaxing solution from the values obtained during maximal $\mathrm{Ca}^{2+}$ activation. $\mathrm{E}_{\mathrm{o}}$ (in $\mathrm{kN} / \mathrm{m}^{2}$ ) was calculated as (adjusted $\Delta$ force/adjusted $\Delta$ length) $\times($ FL/fiber CSA).

Fiber $V_{o} . V_{o}$ was determined from slack-test measurements $(8,22)$. Briefly, the fiber was activated at $\mathrm{pCa} 4.5$ and allowed to attain peak force. The fiber was then subjected to a rapid $(<2 \mathrm{~ms})$ length step so that the fiber became slack and force fell to zero. Force remained at zero while the fiber shortened under no load. Once the fiber had shortened to the point where it was no longer slack, there was a rapid redevelopment of force. The fiber was then transferred back to relaxing solution and reextended to its original FL. The entire process was repeated at a minimum of five different slack lengths that never exceeded $20 \%$ of FL. The times to redevelop force were determined from each record and plotted against the corresponding length steps (Fig. 1). The slope of the best fit line was $V_{0} . V_{0}$ was expressed in FL per second by normalizing shortening velocity to FL at a sarcomere spacing of $2.5 \mu \mathrm{m}$. 


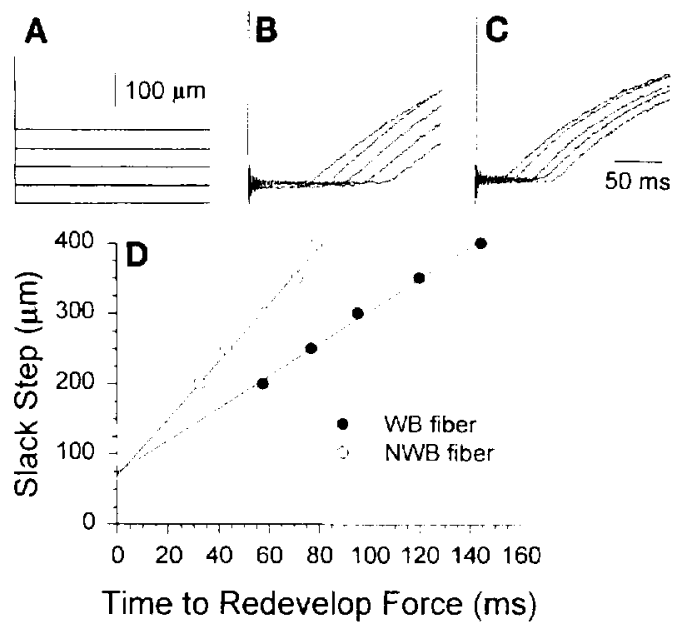

Fig. 1. Determination of maximal shortening velocity $\left(V_{0}\right)$. A: superimposed position records after 5 slack steps ranging from 200 to 400 $\mu \mathrm{m} . B$ and $C$ : corresponding superimposed force records for weightbearing (WB) and non-weight-bearing (NWB) type I soleus fibers, respectively. Note similar time scales. $D$ : time required for redevelopment of force plotted against corresponding slack step. Slope of best fit lines represents $V_{\mathrm{o}}\left(R^{2} \geq 0.99\right.$ for each fiber $)$. When normalized for fiber length (FL), $V_{0}$ values for WB and NWB fibers in this example were 1.13 and $1.67 \mathrm{FL} / \mathrm{s}$, respectively.

MHC determination. After contractile measurements were made, the fiber was removed from the experimental apparatus, solubilized in $10 \mu \mathrm{l}$ of $1 \%$ sodium dodecyl sulfate sample buffer (containing $6 \mathrm{mg} / \mathrm{ml}$ EDTA, $0.06 \mathrm{M}$ tris(hydroxymethyl) aminomethane, $1 \%$ sodium dodecyl sulfate, $2 \mathrm{mg} / \mathrm{ml}$ bromophenol blue, $15 \%$ glycerol, and $5 \% \beta$-mercaptoethanol), and stored at $-80^{\circ} \mathrm{C}$. Later, $0.5 \mathrm{nl}$ of fiber volume was loaded onto a Hoefer SE 600 gel electrophoresis system, consisting of a $3 \%(\mathrm{wt} / \mathrm{vol})$ acrylamide stacking gel and a $5 \%(\mathrm{wt} / \mathrm{vol})$ separating gel, and run at $4^{\circ} \mathrm{C}$ for $22 \mathrm{~h}$. Gels were silver stained according to the procedures described by Giulian et al. (9). A representative gel illustrating MHC identification is presented in Fig. 2. The fibers described in this study contained only the slow type I MHC because these fibers comprise the majority of soleus fibers under both WB and NWB conditions $(8,22,27)$.

Statistical analysis. Data are presented as means $\pm \mathrm{SE}$. Mean values for the WB, NWB, and IWB groups were compared with an analysis of variance. When a significant $F$ ratio was obtained, the Student-Newman-Keuls post hoc procedure was used to locate significant differences among groups. Statistical significance was accepted at $P<0.05$.

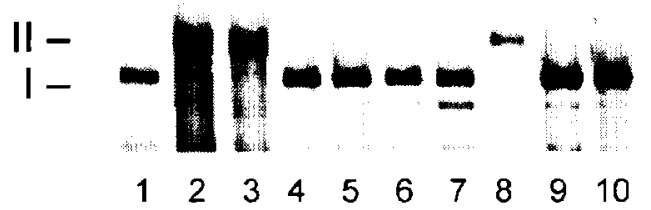

Fig. 2. Representative $5 \%$ polyacrylamide gel illustrating myosin heavy chain composition of soleus fibers. Lanes $1-3$ represent myosin standards prepared from rat soleus (type I), red gastrocnemius (type IIa), and white gastrocnemius (type IIb) muscles, respectively. Lanes 4-10 represent single soleus fibers obtained from intermittent weightbearing (IWB) treatment group. All fibers run on this gel expressed type I myosin heavy chain except for fiber in lane $8 . V_{0}$ values for fibers in lanes $4-10$ were $1.80,1.53,1.59,1.13,3.53,1.68$, and 1.17 FL/s, respectively.

\section{RESULTS}

Effect of NWB. NWB resulted in a significant loss in soleus muscle mass (Table 1). Compared with the WB controls, NWB rats displayed reductions of 40 and $45 \%$ in absolute and relative (muscle mass/body mass) soleus muscle mass, respectively. Consistent with this gross muscle atrophy was a $28 \%$ reduction in the diameter of the type I fibers comprising the soleus (Table 2). Fiber force production was significantly reduced as a result of fiber atrophy, as indicated by a $55 \%$ drop in peak force (Table 2). However, $\mathrm{P}_{\mathrm{o}}$ also fell $16 \%$ after NWB, indicating that atrophied fibers had a reduced ability to produce force per unit CSA. The histogram in Fig. 3 indicates that the majority of the NWB fibers (27 out of 32) displayed $P_{0}$ values that were less than the mean $\mathrm{P}_{0}$ observed for the WB fibers. $\mathrm{E}_{0}$ was reduced by $41 \%$ after $N W B$, whereas the $P_{0} / E_{0}$ ratio increased by $40 \%$ (Table 3 ).

The $V_{0}$ of the WB fibers expressing type I MHC was $1.17 \pm 0.04 \mathrm{FL} / \mathrm{s}$ (Table 4) and ranged from 0.66 to 2.08 FL/s. The average $V_{0}$ of the NWB type I fibers was $33 \%$ higher than the WB mean (Table 4). This increase appeared to be the result of a rightward shift in the $V_{0}$ distribution of the NWB fibers (Fig. 4). For instance, after NWB, $25 \%$ of the type I fibers displayed a $V_{0} \geq$ $1.80 \mathrm{FL} / \mathrm{s}$, whereas no fibers were observed with a $V_{\circ}<$ $1.00 \mathrm{FL} / \mathrm{s}$. This is in contrast to the WB type I $V_{0}$ distribution where only $3 \%$ of the fibers had a $V_{0} \geq 1.80$ $\mathrm{FL} / \mathrm{s}$ and $29 \%$ of the fibers had a $V_{0}<1.00 \mathrm{FL} / \mathrm{s}$.

Effect of IWB. The daily IWB treatments represented $<3 \%$ of the total time spent NWB. This intervention was effective in preventing $22 \%$ of the NWB-induced loss in relative soleus wet weight (Table 1) and $36 \%$ of the decline in type I fiber diameter (Table 2).

IWB was partially effective in restoring force production of type I fibers. Although IWB fibers produced 35\% more peak force than NWB fibers, this was still $39 \%$ less than the force produced by the WB fibers (Table 2). Furthermore, IWB was ineffective in attenuating the decline in $\mathrm{P}_{\mathrm{o}}$ that occurred during 14 days of NWB (Table 2, Fig. 3). Consistent with this observation was the finding that the $E_{o}$ of the IWB fibers remained depressed compared with WB levels and was not statistically different from the NWB value (Table 3 ). The IWB $P_{0} / E_{0}$ was elevated above the WB value and was similar to the value observed for the NWB fibers.

IWB modulated the increase in type I fiber $V_{0}$ that occurred during NWB. The $V_{0}$ of the type I IWB fibers was significantly slower than that of the NWB group

Table 1. Body and soleus weights

\begin{tabular}{lcccl}
\hline \hline Group & $n$ & $\begin{array}{c}\text { Body } \\
\text { Wt, g }\end{array}$ & $\begin{array}{c}\text { Soleus } \\
\text { Wt, mg }\end{array}$ & \multicolumn{1}{c}{$\begin{array}{c}\text { Soleus/Body } \\
\text { Wt, mg/g }\end{array}$} \\
\hline WB & 7 & $353 \pm 6$ & $179 \pm 7$ & $0.51 \pm 0.01$ \\
NWB & 7 & $383 \pm 12$ & $107 \pm 7^{*}$ & $0.28 \pm 0.01^{*}$ \\
IWB & 7 & $355 \pm 12$ & $115 \pm 5^{*}$ & $0.33 \pm 0.01^{*} \dagger$ \\
\hline
\end{tabular}

Values are means $\pm \mathrm{SE}$ of wet wt; $n$, no. of fibers. WB, normal weight bearing; NWB, hindlimb non-weight bearing; IWB, hindlimb NWB with intermittent weight-bearing treatments. ${ }^{*} P<0.05$ vs. WB. $\dagger P<0.05$ vs. NWB. 
Table 2. Diameter, peak force, and peak tension of type I fibers

\begin{tabular}{lllll}
\hline Group & $n$ & $\begin{array}{c}\text { Diameter, } \\
\mu \mathrm{m}\end{array}$ & $\begin{array}{c}\text { Peak Force, } \\
\mathrm{mN}\end{array}$ & $\begin{array}{c}\text { Peak } \\
\text { Tension, } \\
\mathrm{kN} / \mathrm{m}^{2}\end{array}$ \\
\hline WB & 66 & $71 \pm 1$ & $0.51 \pm 0.01$ & $130 \pm 3$ \\
NWB & 32 & $51 \pm 2^{*}$ & $0.23 \pm 0.02^{*}$ & $109 \pm 4^{*}$ \\
IWB & 40 & $58 \pm 1^{* \dagger}$ & $0.31 \pm 0.01^{* \dagger}$ & $117 \pm 3^{*}$ \\
\hline
\end{tabular}

Values are means $\pm \mathrm{SE} . * P<0.05$ vs. WB; $\uparrow P<0.05$ vs. NWB.

but remained elevated above the WB mean (Table 4). Figure 4 illustrates that the IWB $V_{0}$ distribution lacked fibers expressing a relatively high $V_{0}$ because only $5 \%$ of the fibers from this group displayed $V_{\mathrm{o}}$ values that were $\geq 1.80$. However, the IWB mean was still elevated compared with the WB value because only $5 \%$ of the IWB fibers had $V_{0}<1.00 \mathrm{FL} / \mathrm{s}$.

\section{DISCUSSION}

IWB and soleus atrophy. With the removal of normal WB activity, there is a rapid and progressive loss of soleus mass, approaching $20 \%$ after the initial 3 days of NWB (16) and exceeding 50\% of WB control values after 28 days of NWB $(29,31)$. The $45 \%$ reduction in soleus relative wet weight and the $28 \%$ decline in type I fiber diameter noted in the present study are typical responses to 14 days of NWB $(7,8,22,28)$. It is currently thought that this loss of muscle mass is primarily the result of a reduction in the tonic WB contractile activity normally performed by the soleus in a $1-G$ environment $(7,26,28,31)$. The reduced working length of the soleus, resulting from chronic plantar
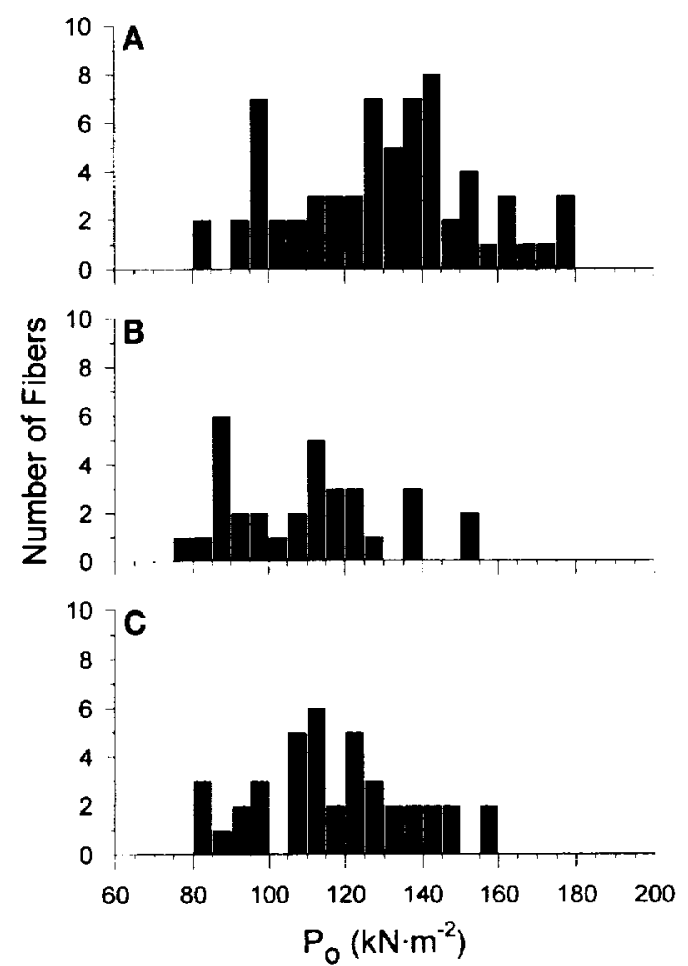

Fig. 3. Frequency distributions of peak tension $\left(\mathrm{P}_{0}\right)$ for WB $(A)$, NWB $(B)$, and IWB $(C)$ type I fibers.
Table 3. Peak elastic modulus of type I fibers

\begin{tabular}{lccc}
\hline \hline Group & $n$ & $\begin{array}{c}\mathrm{E}_{0}, \\
\times 10^{4} \mathrm{kN} / \mathrm{m}^{2}\end{array}$ & $\mathrm{P}_{\mathrm{o}} / \mathrm{E}_{\mathrm{o}}$ \\
\hline WB & 25 & $2.09 \pm 0.09$ & $60 \pm 2$ \\
NWB & 12 & $1.24 \pm 0.08^{*}$ & $84 \pm 4^{*}$ \\
IWB & 23 & $1.44 \pm 0.05^{*}$ & $76 \pm 3^{*}$ \\
\hline
\end{tabular}

Values are means $\pm S E$. $E_{o}$, peak elastic modulus; $P_{o}$, peak isometric tension. ${ }^{*} P<0.05$ vs. WB.

flexion during NWB, may contribute to the atrophic response (28). It is not surprising that IWB would be an effective countermeasure to NWB soleus atrophy because it most likely reverses these conditions. However, what is noteworthy is the relatively brief amount of WB activity required to attenuate, or even to prevent, soleus atrophy during short-term periods of NWB. A total of 40-60 min of normal WB activity per day, or $3-4 \%$ of the total NWB time, is sufficient to maintain soleus mass at WB control levels during a 7-day period of hindlimb suspension $(3,13,26)$.

In contrast, the IWB treatments in the present study attenuated only a relatively small portion $(22 \%)$ of the soleus atrophy that occurred during NWB. The most likely explanation for this difference is that intermittent weight support is considerably less effective in preserving soleus muscle mass over the 14 days of NWB examined in the present study, compared with the 7-day periods of NWB investigated by others. This reduced effectiveness may be related to changes in the rates of myofibril protein synthesis and degradation that occur during hindlimb suspension. The soleus myofibril protein synthesis rate declines to a new steady-state level within the first few days of hindlimb suspension, but myofibril protein degradation does not reach its maximum rate until days 9-15 (30). Consequently, the net fractional rate of soleus myofibril protein loss peaks on day 15 of NWB (30). It seems likely that the stimulus provided by IWB is sufficient to preserve soleus mass during the first week of hindlimb suspension but is less effective in preventing soleus atrophy as the rate of protein loss rises during the second week of NWB. This interpretation is also consistent with the observation that daily 2- to 4 -h periods of WB during 28 days of hindlimb suspension attenuate soleus atrophy to a greater degree (50\%) than observed in the present study because a new steady state of myofibril protein metabolism has been established in the NWB soleus by that time $(30,31)$.

IWB and fiber force. Both spaceflight and hindlimb suspension are known to reduce the ability of the rodent soleus to produce force $(1,5,7,29,33)$. Studies

Table 4. Maximal shortening velocity of type I fibers

\begin{tabular}{lcl}
\hline \hline Group & $n$ & \multicolumn{1}{c}{$\mathrm{V}_{\mathrm{o}}, \mathrm{FL}_{\mathrm{s}}{ }^{1}$} \\
\hline WB & 66 & $1.17 \pm 0.04$ \\
NWB & 32 & $1.56 \pm 0.06^{*}$ \\
IWB & 40 & $1.37 \pm 0.05^{*+}$ \\
\hline
\end{tabular}

Values are means \pm SE, $V_{0}$ maximal shortening velocity; FL, fiber length. ${ }^{*} P<0.05$ vs. WB; $+P<0.05$ vs. NWB. 


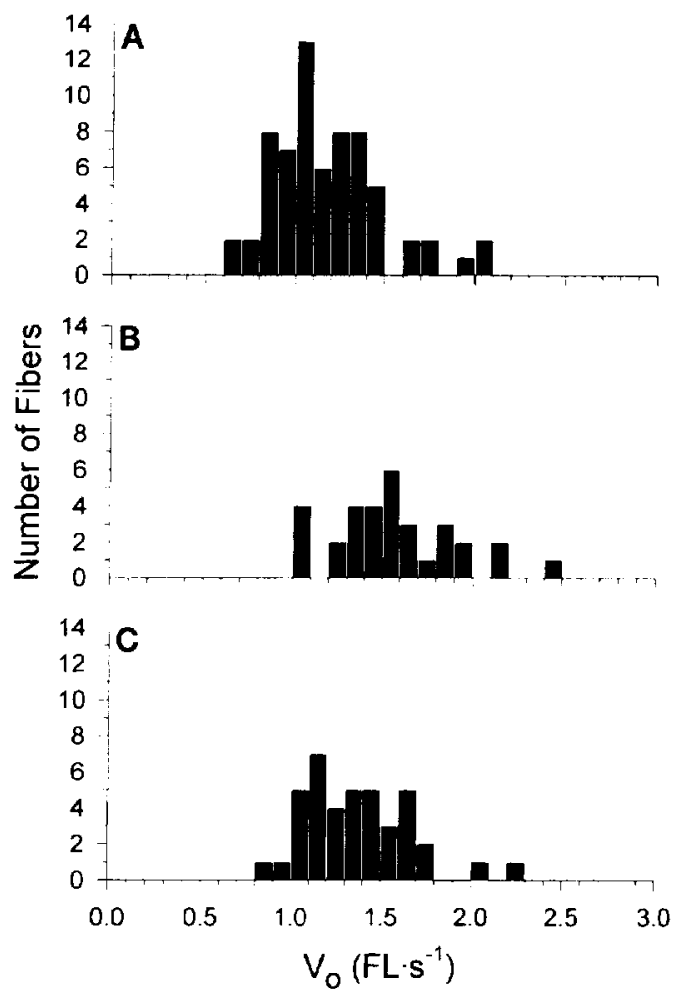

Fig. 4. Frequency distributions of maximal $V_{0}$ for WB $(A)$, NWB $(B)$, and IWB $(C)$.

performed on single soleus fibers indicate that the reduction in soleus force production during NWB is due not only to gross fiber atrophy but also to a reduction in $P_{0}$, or the force produced per CSA of fiber $(8,21,22,27)$. The recent observation that fiber $E_{0}$ is also reduced after 7-21 days of NWB suggests that the NWBinduced reduction in $\mathrm{P}_{0}$ is due to a decline in the number of cross bridges per fiber $\operatorname{CSA}(23)$. This finding is consistent with the disproportionate loss of myofibril protein compared with the overall loss of muscle mass that occurs during hindlimb suspension (32).

The 55\% decline in force, $16 \%$ decline in $\mathrm{P}_{0}$, and $41 \%$ reduction in $\mathrm{E}_{\mathrm{o}}$ observed for the NWB fibers in the present study confirm these previous conclusions. More importantly, the present data indicate that a modest intervention of $40 \mathrm{~min} /$ day of standing WB has differential effects on the two mechanisms responsible for the loss of force during NWB. For instance, IWB increased type I fiber peak isometric force by $35 \%$ over the NWB condition. This partial restoration of force production was primarily due to a reduction in the degree of fiber atrophy experienced by the IWB fibers. In contrast, $\mathrm{P}_{0}$ and $E_{0}$ were not statistically different from the NWB condition, indicating that the IWB treatments had no affect on NWB-induced alterations in the force produced per fiber CSA or on the number of cross bridges per fiber CSA, respectively. Therefore, fibers from both the NWB and IWB groups lost cross bridges at a rate that was disproportionate to their degree of fiber atrophy, even though the IWB treatment effectively slowed the rate of fiber atrophy and thereby partially restored force production. These data further suggest that the mechanisms responsible for the loss of force during NWB have different degrees of responsiveness to countermeasure intervention because a stimulus that effectively reduced the degree of fiber atrophy had no effect on a decline in the number of cross bridges per fiber CSA.

The reduced number of cross-bridge attachments per CSA after IWB is likely to have important functional consequences. At the whole muscle level, one would expect to observe a reduction in the force produced per CSA or per unit mass of the soleus after this treatment. However, it has been reported that IWB maintained soleus peak isometric force per unit mass during 7 days of hindlimb suspension (26). The discrepancy between these findings and the present results could be due to the different durations of NWB as noted above.

The increase in $\mathrm{P}_{0} / \mathrm{E}_{0}$ after NWB is consistent with previous results from this laboratory (23). McDonald and Fitts (23) point out that an increase in this ratio after NWB could represent either an increase in the average force per cross-bridge attachment, a decrease in fiber stiffness, or a disproportionate change in these variables. These authors hypothesized that the increased $P_{o} / E_{o}$ after NWB resulted from the loss of contractile protein that, in turn, increased the filament lattice spacing of the atrophied fibers. An increase in lattice spacing has been shown to decrease both fiber force and stiffness, with the effect on stiffness quantitatively greater (12). Consequently, the $\mathrm{P}_{0} / \mathrm{E}_{0}$ would increase. If this hypothesis is correct, the NWB-induced decline in $\mathrm{P}_{0}$ would result from fewer cross bridges per fiber CSA and to a reduced force per cross bridge. Interestingly, an increase in the spacing between thin and thick myofilaments also results in an increase in fiber $V_{\mathrm{o}}$ and myosin adenosinetriphosphatase activity $(19,24)$, a rightward shift of the force-pCa relationship (11), and a reduction in force per fiber $\operatorname{CSA}(11,24)$, all of which have been observed in skinned type I fibers after NWB $(8,21,22,27)$.

The increase in $\mathrm{P}_{0} / \mathrm{E}_{\mathrm{o}}$ after NWB was not prevented by the IWB treatments in this study. The simplest interpretation of this data is that the IWB treatments had no effect on those factors leading to an increased $\mathrm{P}_{0} / \mathrm{E}_{0}$ in the NWB condition that, on the basis of the interpretation by McDonald and Fitts (23), would suggest that the IWB treatments had no effect on proposed filament lattice-spacing changes that may occur during NWB. An alternative interpretation is that NWB and/or IWB resulted in an increase in the apparent average force per actomyosin cross bridge. This interpretation seems less likely because the increase in force per cross-bridge attachment would have to approach $40 \%$.

$I W B$ and fiber $V_{o}$. We observed a $33 \%$ increase in the $V_{0}$ of single fibers expressing type I MHC after 14 days of NWB, a change that is in good agreement with previous observations $(8,21,22,27)$. Although the mechanism responsible has not been identified, the elevated $V_{0}$ does not appear to be due to undetected levels of fast MHC in single fibers classified as type I or to changes in myosin light-chain stoichiometry $(21,22)$. In the present study, IWB reduced, but did not completely prevent, this NWB-induced increase in type I 
$V_{0}$. Interestingly, this finding appears to be the result of a reduction in the number of fibers expressing a relatively high $V_{0}$ (i.e., $V_{o}>1.80 \mathrm{FL} / \mathrm{s}$ ) coupled with little restoration in the number of fibers expressing relatively low $V_{\mathrm{o}}$ (i.e., $V_{\mathrm{o}}<1.00 \mathrm{FL} / \mathrm{s}$ ). Because almost $30 \%$ of the type I WB population expressed $V_{0}$ values $<1.00 \mathrm{FL} / \mathrm{s}$, the overall mean $V_{0}$ of the IWB group remained elevated compared with the WB condition. It is known that soleus muscle activity shifts from a tonic to a phasic pattern during hindlimb suspension (28). If that same pattern is maintained during the periods of IWB, those fibers with a relatively high $V_{0}$ may be recruited to a greater extent during standing and may thereby display a greater adaptation to the IWB treatment. However, this mechanism remains speculative because it is impossible to determine from the present data whether the reduced number of high $V_{\mathrm{o}}$ fibers represent a reduction in $V_{0}$ of a specific population of fibers, with little change in the remaining nonresponsive fibers, or whether all fibers in the IWB fiber population simply shifted toward lower velocities.

Summary and recommendations. To summarize, IWB treatments that totaled $<3 \%$ of the entire time spent NWB appear to be considerably less effective in preventing soleus muscle atrophy over a period of 14 days than previously observed for shorter periods of NWB. Nevertheless, this treatment reduced soleus muscle atrophy and attenuated (but did not prevent) alterations in soleus type I fiber diameter, peak force production, and $V_{0}$. In contrast, IWB had little effect on NWB-induced reductions in type I fiber $P_{0}$ and $E_{0}$, which suggests that reductions in actomyosin cross-bridge attachments per fiber CSA are more resistant to countermeasure intervention.

The inability of the IWB treatment to prevent changes in single-fiber contractile function over 14 days of NWB is a shortcoming of this countermeasure. It remains to be determined whether normal fiber function could be maintained with a modified IWB protocol that incorporates changes in the duration and/or the number of daily WB sessions. Previous work suggests that it is the number of sessions each day, rather than the duration of each session, that is the primary factor in determining the effectiveness of IWB as a countermeasure (3). An alternative to IWB would be to incorporate more forceful muscle contractions into the countermeasure treatment because recent studies have demonstrated the effectiveness of high-intensity resistance exercise in maintaining soleus mass and muscle tension during $7-10$ days of NWB $(14,18)$.

This study was supported by National Aeronautics and Space Administration Postdoctoral Space Biology Research Associate Award SBRA93-06 to J. J. Widrick and by Grants NAG2-212 and NAGW4376 to R. H. Fitts, as well as by a Wisconsin Space Grant Consortium undergraduate scholarship to J. J. Bangart.

Address for reprint requests: R. H. Fitts, Marquette Univ., Dept. of Biology, Wehr Life Sciences Bldg., Rm. 109, PO Box 1881, Milwaukee, WI 53201-1881.

Received 20 March 1995; accepted in final form 27 October 1995.

\section{REFERENCES}

1. Caiozzo, V. J., M. J. Baker, R. E. Herrick, M. Tao, and K. M. Baldwin. Effect of spaceflight on skeletal muscle: mechanical properties and myosin isoform content of a slow muscle. J. Appl. Physiol. 76: 1764-1773, 1994.

2. Convertino, V.A. Physiological adaptations to weightlessness: effects on exercise and work performance. In: Exercise and Sport Sciences Reviews, edited by K. B. Pandolf and J. O. Holloszy. Baltimore, MD: Williams \& Wilkins, 1990, vol. 18, p. 119-166.

3. D'Aunno, D. S., R. R. Robinson, G. S. Smith, D. B. ThomaBon, and F. W. Booth. Intermittent acceleration as a countermeasure to soleus muscle atrophy. J. Appl. Physiol. 72: 428-433, 1992.

4. D'Aunno, D. S., D. B. Thomason, and F. W. Booth. Centrifugal intensity and duration as countermeasures to soleus muscle atrophy. J. Appl. Physiol. 69: 1387-1389, 1990.

5. Diffee, G. M., V. J. Caiozzo, R. E. Herrick, and K. M. Baldwin. Contractile and biochemical properties of rat soleus and plantaris after hindlimb suspension. Am. J. Physiol. 260 (Cell Physiol. 29): C528-C534, 1991.

6. Fabiato, A., and F. Fabiato. Calculator programs for computing the composition of the solutions containing multiple metals and ligands used for experiments in skinned muscle cells. $J$. Physiol. Paris 75: 463-505, 1979.

7. Fitts, R. H., J. M. Metzger, D. A. Riley, and B. R. Unsworth. Models of disuse: a comparison of hindlimb suspension and immobilization. J. Appl. Physiol. 60: 1946-1953, 1986.

8. Gardetto, P. R., J. M. Schluter, and R. H. Fitts. Contractile function of single muscle fibers after hindlimb suspension. $J$. Appl. Physiol. 66: 2739-2749, 1989.

9. Giulian, G. G., R. L. Moss, and M. Greaser. Improved methodology for analysis and quantitation of proteins on onedimensional silver-stained slab gels. Anal. Biochem. 129: 277287, 1983.

10. Godt, R. E., and B. D. Lindley. Influence of temperature upon contractile activation and isometric force production in mechanically skinned muscle fibers of the frog. J. Gen. Physiol. 80: 279-297, 1982 .

11. Godt, R. E., and D. W. Maughan. Influence of osmotic compression on calcium activation and tension in skinned muscle fibers of the rabbit. Pfluegers Arch. 391: 334-337, 1981.

12. Goldman, Y. E., and R. M. Simmons. The stiffness of frog skinned muscle fibres at altered lateral filament spacing. $J$. Physiol. Lond. 378: 175-194, 1986.

13. Hauschka, E. O., R. R. Roy, and V. R. Edgerton. Periodic weight support effects on rat soleus fibers after hindlimb suspension. J. Appl. Physiol. 65: 1231-1237, 1988.

14. Herbert, M. E., R. R. Roy, and V. R. Edgerton. Influence of one-week hindlimb suspension and intermittent high load exercise on rat muscles. Exp. Neurol. 102: 190-198, 1988.

15. Huxley, A. F., and R. M. Simmons. Proposed mechanism of force generation in striated muscle. Nature Lond. 233: 533-538, 1971.

16. Jaspers, S. R., and M. E. Tischler. Atrophy and growth failure of rat hindlimb muscles in tail-cast suspension. J. Appl. Physiol. 57: 1472-1479, 1984.

17. Jiang, B., R. R. Roy, C. Navarro, and V. R. Edgerton. Absence of a growth hormone effect on rat soleus atrophy during a 4-day spaceflight. J. Appl. Physiol. 74: 527-531, 1993.

18. Kirby, C. R., M. J. Ryan, and F. W. Booth. Eccentric exercise training as a countermeasure to non-weight-bearing soleus muscle atrophy. J. Appl. Physiol. 73: 1894-1899, 1992.

19. Krasner, B., and D. Maughan. The relationship between ATP hydrolysis and active force in compressed and swollen skinned muscle fibers of the rabbit. Pfluegers Arch. 400: 160-165, 1984.

20. Martin, T. P., V. R. Edgerton, and R. E. Grindeland. Influence of spaceflight on rat skeletal muscle. J. Appl. Physiol. 65: 2318-2325, 1988.

21. McDonald, K. S., C. A. Blaser, and R. H. Fitts. Force-velocity and power characteristics of rat soleus muscle fibers after hindlimb suspension. J. Appl. Physiol. 77: 1609-1616, 1994.

22. McDonald, K. S., and R. H. Fitts. Effect of hindlimb unweighting on single soleus fiber maximal shortening velocity and ATPase activity. J. Appl. Physiol. 74: 2949-2957, 1993.

23. McDonald, K. S., and R. H. Fitts. Effect of hindlimb unloading on rat soleus fiber force, stiffness, and calcium sensitivity. $J$. Appl. Physiol. 79: 1796-1802, 1995. 
24. Metzger, J. M., and R. L. Moss. Shortening velocity in skinned single muscle fibers. Influence of filament lattice spacing. Biophys. J. 52: 127-131, 1987.

25. Moss, R. L. Sarcomere length-tension relations of frog skinned muscle fibres during calcium activation at short lengths. $J$. Physiol. Lond. 292: 177-192, 1979.

26. Pierotti, D. J., R. R. Roy, V. Flores, and V. R. Edgerton. Influence of 7 days of hindlimb suspension and intermittent weight support on rat muscle mechanical properties. Aviat. Space Environ. Med. 61: 205-210, 1990.

27. Reiser, P. J., C. E. Kasper, and R. L. Moss. Myosin subunits and contractile properties of single fibers from hypokinetic rat muscles. J. Appl. Physiol. 63: 2293-2300, 1987.

28. Riley, D. A., G. R. Slocum, J. L. W. Bain, F. R. Sedlak, T. E. Sowa, and J. W. Mellender. Rat hindlimb unloading: soleus histochemistry, ultrastructure, and electromyography. J. Appl. Physiol. 69: 58-66, 1990.
29. Templeton, G. H., M. Padalino, J. Manton, M. Glasberg, C. J. Silver, P. Silver, G. DeMartino, T. Leconey, G. Klug, H. Hagler, and J. L. Sutko. Influence of suspension hypokinesia on rat soleus muscle. J. Appl. Physiol. 56: 278-286, 1984.

30. Thomason, D. B., R. B. Biggs, and F. W. Booth. Protein metabolism and $\beta$-myosin heavy-chain mRNA in unweighted soleus muscle. Am. J. Physiol. 257 (Regulatory Integrative Comp. Physiol. 26): R300-R305, 1989.

31. Thomason, D. B., R. E. Herrick, and K. M. Baldwin. Activity influences on soleus muscle myosin during rodent hindlimb suspension. J. Appl. Physiol. 63: 138-144, 1987.

32. Thomason, D. B., R. E. Herrick, D. Surdyka, and K. M. Baldwin. Time course of soleus muscle myosin expression during hindlimb suspension and recovery. J. Appl. Physiol. 63: 130-137, 1987.

33. Winiarski, A. M., R. R. Roy, E. K. Alford, P. C. Chiang, and V. R. Edgerton. Mechanical properties of rat skeletal muscle after hind limb suspension. Exp. Neurol. 96: 650-660, 1987.

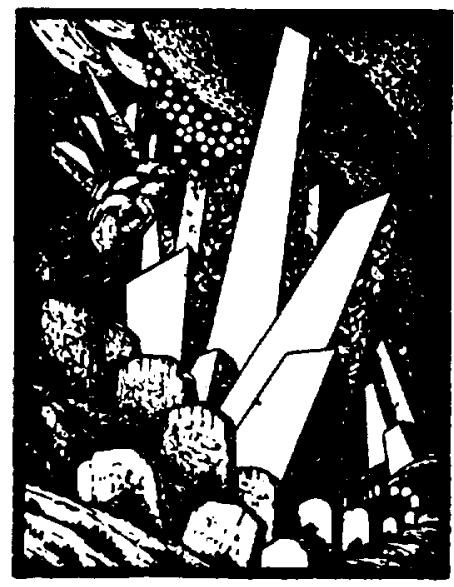


